Петрик М. Р., д.ф.-м.н., професор (Тернопільський національний технічний університет ім. Івана Пулюя, м. Тернопіль), Хіміч 0. М., чл.-кор. НАНУ, д.ф.-м.н., професор (Інститут кібернетики ім. В.М. Глушкова НАНУ, м. Київ), Бойко І. В., к.ф.-м.н., доцент, Петрик О. Ю. (Тернопільський національний технічний університет ім. Івана Пулюя, м. Тернопіль)

\title{
ВИСОКОПРОДУКТИВНІ ОБЧИСЛЮВАЛЬНІ МЕТОДИ І ТЕХНОЛОГІЇ МОДЕЛЮВАННЯ НАНОПОРИСТИХ СИСТЕМ ЗІ ЗВОРОТНИМИ ЗВ'ЯЗКАМИ ДЛЯ АДСОРБЦІЙНОГО ОЧИЩЕННЯ ГАЗІВ
}

Розглядається задача моделювання неізотермічної адсорбції та десорбції в нанопористих цеоліт-системах для нелінійної адсорбційної рівноваги типу Ленгмюра. Пропонуються висопродуктивні методи операційного числення Гевісайда та перетворення Лапласа 3 використанням схем лінеаризації на основі підходу Ландау щодо розкладу в ряд функції рівноваги Ленгмюра в околі точки температури фазового переходу та малого параметру, що дозволяють ефективне розпаралелювання обчислень. Подані результати обчислювальних експериментів.

Ключові слова: високопродуктивні обчислення, нанопористі системи зі зворотними зв'язками, адсорбція та десорбція газів, функція адсорбційної рівноваги Ленгмюра, операційний метод Гевісайда, інтегральне перетворення Лапласа.

Вступ. Впровадження сучасних кіберфізичних нанопористих систем поглинання шкідливих викидів вуглецевих сполук об'єктів енергетики і транспорту - ключ до вирішення світової проблеми глобального потепління, покращання стану здоров'я людини, довкілля та реалізації стратегії безпечної енергетики [1]. Якість математичних моделей процесів адсорбції та десорбції вуглеводнів у нанопористих каталітичних середовищах та високопродуктивних методів побудови їх розв'язків з урахуванням архітектури сучасних обчислювальних систем визначає ефективність технологічних рішень для нейтралізації та зменшення викидів відпрацьованих газів, що стрімко зростає і сприяє глобальному потеплінню [2].

В даний час проводяться численні експериментальні та теоретичні дослідження таких процесів, особливо дослідження щодо удо- 
сконалення їх математичних моделей з урахуванням впливу різних лімітуючих чинників внутрішньої кінетики адсорбції, десорбції та тепломасопереносу мікропористих середовищах [3-5].

В цій праці, що $є$ розиненням робіт [6-11] окреслені теоретичні основи моделювання неізотермічної адсорбції та десорбції в нанопористих каталізаторах для нелінійної ізотерми Ленгмюра, яка найповніше визначає механізм адсорбційної рівноваги для нанопористих цеоліт-систем. Для моделювання використовуються високопродуктивні методи операційного числення Гевісайда та інтегрального перетворення Лапласа з використанням схем лінеаризації на основі підходу Ландау щодо розкладу в ряд неізотермічної функції рівноваги в околі точки температури фазового переходу та обгрунтування малого параметру, що дозволило отримувати високошвидкісні аналітичні розв'язки, що підвищують якість розпаралелювання обчислювального процесу і моделювання в цілому.

1. Нелінійна математична модель неізотермичних адсорбції та десорбції на основі узагальненого рівняння адсорбційної рівноваги Ленґмюра

Вхідний потік газової суміші дифундує в макропорах (межчастинковому простірі) каталітичного середовища і в нанопорах частинок (кристалітів) цеоліту. Загальна гіпотеза, прийнята в розробці моделі, полягає в тому, що адсорбційна взаємодія молекул з активними центрами адсорбції на поверхні фазового розділення в нанопорах кристалітів визначається на основі нелінійного рівняння адсорбційної рівноваги, отриманим Нобелівським лавреатом Е. Ленґмюром 3 урахуванням наступних фізичних припущень [12; 13]:

1. Адсорбція локалізована і обумовлена силами, близькими до хімічних сил: силами дисперсії, взаємодія яких встановлюється законом Джона-Ленарда та електростатичними силами тяжіння та відштовхування, механізм дії яких описаний Ван-Дер-Ваальсом [13] .

2. Адсорбція відбувається в активних центрах на поверхні адсорбенту та розподілена по всій внутрішній поверхні мікро- та нанопор. Кожний активний центр адсорбує лише одну молекулу адсорбату, а їі молекулярний шар адсорбату формується на поверхні.

3. Адсорбовані молекули зберігаються активними центрами протягом певного час, залежно від температури.

Виходячи з цього, функція адсорбційної рівноваги типу Ленґмюра, що описує фазовий адаптивний перехід із середовища потоку газу до нанопор шару адсорбції, буде визначатися нелінійною взаємодією, що встановлює зв'язок між рівноважною концентрацією $c_{e q}$ 


$$
a\left(c_{e q}\right)=a_{\text {full }} \frac{b(T) c_{e q}}{1+b c_{e q}} .
$$

Тут $\Delta H$ - енергія активації, кДж/моль; $R$ - газова константа, кДж/моль/(м $\left.{ }^{3}\right) ; a_{\text {full }}, 0<b_{0}<1, b_{0}$ - емпіричні коефіцієнти, які залежать від властивостей мікропористих адсорбентів та компонентів асорбату ( $b_{0}$ рівний співвідношенню констант швидкості десорбції та адсорбції), $a_{\text {full }}$ - концентрація адсорбату в мікропорах цеоліту з повним заповненням адсорбційних центрів, моль/г.

Енергія активації, що має фізичний зміст у цій задачі як теплота адсорбції, визначається як:

$$
\Delta H=\bar{\varphi}-\left(U_{g}-U_{s}\right)-R T,
$$

де $U_{g}-U_{s}$ різниця між кінетичною енергією молекули адсорбату в газоподібних та адсорбованих станах залежно від природи адсорбованої фази, $\bar{\varphi}$ - це величина потенціалу Ленарда-Джонса, усереднена по об'єму пор адсорбенту [13], і є характеристикою цеоліту.

Крім того, оскільки $U_{g}=\frac{i_{g}}{2} R T ; U_{s}=\frac{i_{s}}{2} R T$, де $i_{g}, i_{s}$ - число ступенів свободи молекул адсорбату у відповідній фазі. Тоді, використовуючи принцип рівномірного розподілу енергії в ступенях свободи, маємо

$$
U_{g}-U_{s}=\frac{\left(i_{g}-i_{s}\right)}{2} R T .
$$

Потенціал Ленарда-Джонса визначається як [12]:

$$
\phi(r)=4 \tilde{\varepsilon}\left[\left(\frac{r_{0}}{r}\right)^{12}-\left(\frac{r_{0}}{r}\right)^{6}\right],
$$

де параметри $\tilde{\varepsilon}$ і $r$ залежать від природи молекул адсорбату.

Отримавши з (1) рівноважну концентраційну функцію $c_{e q}$ щодо концентрації адсорбату в нанопорах а, отримаємо

$$
c_{e q}(a)=\frac{1}{b(T)} \frac{a}{a_{\text {full }}-a} .
$$

Остаточно кінетика неізотермічної адсорбції та десорбції в нанопористих цеолітах з урахуванням нелінійної функції адсорбційної рівноваги та даних фізичних обґрунтувань описується наступною системою нелінійних рівнянь в частинних похідних $[11 ; 14]$ : 


$$
\begin{gathered}
\frac{\partial c(t, z)}{\partial t}+\frac{\partial a(t, z)}{\partial t}+u \frac{\partial c}{\partial z}=D_{\text {inter }} \frac{\partial^{2} c}{\partial z^{2}}, \\
-H \frac{\partial T(t, z)}{\partial t}-u h_{g} \frac{\partial T}{\partial z}-Q \frac{\partial a}{\partial t}-\mathrm{X}^{2} T+\Lambda \frac{\partial^{2} T}{\partial z^{2}}=0, \\
\frac{\partial a}{\partial t}=\beta\left(c-\frac{1}{b_{0} \exp \left(-\frac{\Delta H}{R T}\right)} \frac{a}{a_{\text {full }}-a}\right) .
\end{gathered}
$$

Початкові умови:
а) адсорбція:
b) десорбція:

$$
\begin{array}{ll}
\left.c(t, z)\right|_{t=o}=0, & \left.c(t, z)\right|_{t=o}=c_{0}^{0}, \\
\left.T(t, z)\right|_{t=o}=T_{0}^{0}, & \left.T(t, z)\right|_{t=o}=T_{0}^{0},
\end{array}
$$

Крайові умови:

$$
\begin{array}{cc}
\text { a) адсорбція: } & \text { b) десорбція: } \\
\left.c(t, z)\right|_{z=o}=c_{i n}, & \left.c(t, z)\right|_{z=0}=c_{i n}(t), \\
\left.\frac{\partial}{\partial z} c(t, z)\right|_{z=\infty}=0, & \left.\frac{\partial}{\partial z} c(t, z)\right|_{z=\infty}=0, \\
\left.T(t, z)\right|_{z=0}=T_{i n},\left.\frac{\partial}{\partial z} T(t, z)\right|_{z=\infty}=0, & \left.T(t, z)\right|_{z=0}=T_{i n}(t),\left.\frac{\partial}{\partial z} T(t, z)\right|_{z=\infty}=0 .
\end{array}
$$

Схема лінеаризації нелінійної моделі. Виконаємо деякі розклади в ряд елементів нелінійної компоненти $\frac{1}{\exp \left(-\frac{\Delta H}{R T}\right)} \frac{a}{a_{\text {full }}-a}$ диференціального рівняння (7). Розклавши згідно підходу Ландау вираз $\exp \left(\frac{\Delta H}{R T}\right)$ в ряд Тейлора в околі точки температури фазового переходу $T_{e q}$, що відповідає положенню рівноваги «адсорбатмікропори адсорбенту», отримуємо [15] :

$$
\begin{array}{r}
\exp \left(\frac{\Delta H}{R T}\right)=\exp \left(\frac{\Delta H}{R T_{e q}}\right)-\frac{\Delta H}{R} \exp \left(\frac{\Delta H}{R T_{e q}}\right) \frac{1}{T_{e q}^{2}}\left(T-T_{e q}\right)+\frac{1}{2 !}\left(\frac{\Delta H}{R}\right)^{2} \exp \left(\frac{\Delta H}{R T_{e q}}\right)\left(\frac{1}{T_{e q}^{4}}+\frac{R}{2 \Delta H} \frac{1}{T_{4 e q}^{3}}\right)\left(T-T_{e q}\right)^{2}+\ldots \\
3 \\
\text { урахуванням того, що величина } \frac{a}{a_{\text {full }}}<1 \text {, вираз } \frac{a / a_{\text {full }}}{1-\frac{a}{a_{\text {full }}}} \text { роз- }
\end{array}
$$
кладаєм в ряд Маклорена [16]: 


$$
\frac{a / a_{\text {full }}}{1-\frac{a}{a_{\text {full }}}}=\frac{1}{1-\frac{a}{a_{\text {full }}}}-1=\frac{a}{a_{\text {full }}}+\left(\frac{a}{a_{\text {full }}}\right)^{2}+\ldots+\left(\frac{a}{a_{\text {full }}}\right)^{n}+\ldots
$$

Нехтуючи членами не менше другого порядку у формулах (13), (14), вираз $\frac{1}{\exp \left(-\frac{\Delta H}{R T}\right)} \frac{a}{a_{\text {full }}-a}$ набуде вигляду [11]:

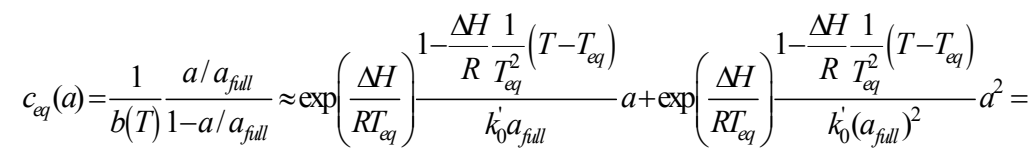
$=\frac{1+\frac{\Delta H}{R T_{e q}}}{k_{0}^{\prime} a_{\text {full }}} \exp \left(\frac{\Delta H}{R T_{e q}}\right) a+\frac{1+\frac{\Delta H}{R T_{e q}}}{k_{0}^{\prime}\left(a_{\text {full }}\right)^{2}} \exp \left(\frac{\Delta H}{R T_{e q}}\right) a^{2}-\frac{1}{k_{0}^{\prime} a_{\text {full }}} \frac{\Delta H}{R} \frac{1}{T_{e q}^{2}} \exp \left(\frac{\Delta H}{R T_{e q}}\right) a T-\frac{1}{k_{0}^{\prime}\left(a_{\text {full }}\right)^{2}} \frac{\Delta H}{R} \frac{1}{T_{e q}^{2}} \exp \left(\frac{\Delta H}{R T_{e q}}\right) T a^{2} \approx$ $\approx \gamma a(t, z)+\varepsilon a^{2}(t, z)-\varepsilon \theta \varepsilon a(t, z) T(t, z)$, де $\gamma=\frac{1+\frac{\Delta H}{R}}{k_{0}^{\prime} a_{\text {full }}} \exp \left(\frac{\Delta H}{R T_{e q}}\right)$ - константа адсорції, що описує лінійну компоненту функції адсорбційної рівноваги $c_{e q}(a)$ (згідно закону Генрі), $\varepsilon=\frac{1+\frac{\Delta H}{R}}{k_{0}^{\prime}\left(a_{f u l l}\right)^{2}} \exp \left(\frac{\Delta H}{R T_{e q}}\right)$ - малий параметр, що враховує нелінійну компоненту неізотерми адсорбції, $\theta=\frac{a_{\text {full }}}{1+\frac{\Delta H}{R}} \frac{\Delta H}{R} \frac{1}{T_{e q}^{2}}, Q=\frac{\Delta H}{M_{\text {ads }}}-$ теплота адсорбції.

Підставляючи розкладений вираз (15) замість залежності $c_{e q}(a)$ в третьому рівнянні системи (5)-(7), отримаємо

$$
\frac{\partial a}{\partial t}=\beta(c-\gamma a(z, t)-\varepsilon a(z, t)(a(t, z)-\theta T(t, z)) .
$$

2. Методика побудови аналітичних розв'язків системи неоднородних крайових задач адсорбції/десорбції

Лінеаризована система задач з малим параметром. Задача (5)(12) з урахуванням апроксимаційного кінетичного рівняння фазового перетворення (16), що містить малий параметр $\varepsilon, \epsilon$ змішаною крайовою задачею для нелінійної системи диференціальних рівнянь в частинних похідних другого порядку. Розв'язок задачі (5)-(12) шукаємо за допомогою асимптотичних розкладу за малим параметром $\varepsilon$ у ви70 
гляді наступних степенних рядів [11]:

$$
\begin{gathered}
c(t, \mathrm{z})=c_{0}(t, \mathrm{z})+\varepsilon c_{1}(t, \mathrm{z})+\varepsilon^{2} c_{2}(t, \mathrm{z})+\ldots, \\
T(t, \mathrm{z})=T_{0}(t, \mathrm{z})+\varepsilon T_{1}(t, \mathrm{z})+\varepsilon^{2} T_{2}(t, \mathrm{z})+\ldots, \\
a(t, \mathrm{z})=a_{0}(t, \mathrm{z})+\varepsilon a_{1}(t, \mathrm{z})+\varepsilon^{2} a_{2}(t, \mathrm{z})+\ldots
\end{gathered}
$$

В результаті підстановки асимптотичних сум (17) у рівняння (5)(12), вихідна нелінійна крайова задача (5)-(12) розпаралелюється на два типи лінеаризованих крайових задач [10; 11]:

Задача $A_{0}$ (нульове наближення з початковими та крайовими умовами вихідної нелінійної задачі): знайти розв'язок в області $D=\{(t, \mathrm{z}): t>0, \quad z \in(0, \infty)\}$ системи рівнянь в частинних похідних:

$$
\begin{gathered}
\frac{\partial c_{0}(t, \mathrm{z})}{\partial t}+\frac{\partial a_{0}(t, \mathrm{z})}{\partial t}+u \frac{\partial c_{0}}{\partial x}=D_{\text {inter }} \frac{\partial^{2} c_{0}}{\partial z^{2}} \\
-H \frac{\partial T_{0}(t, z)}{\partial t}-u h_{g} \frac{\partial T_{0}}{\partial z}-Q \frac{\partial a_{0}}{\partial t}-X^{2} T_{0}+\Lambda \frac{\partial^{2} T_{0}}{\partial z^{2}}=0 \\
\frac{\partial a_{0}}{\partial t}=\beta\left(c_{0}-\gamma a_{0}\right) .
\end{gathered}
$$

Початкові умови:

а) адсорбція:

b) десорбція:

$$
\begin{array}{ll}
\left.c_{0}(t, z)\right|_{t=o}=0, & \left.c_{0}(t, z)\right|_{t=o}=c_{0}^{0}, \\
\left.T_{0}(t, z)\right|_{t=o}=T_{0}^{0}, & \left.T_{0}(t, z)\right|_{t=o}=T_{0}^{0},
\end{array}
$$

Крайові умови:

$$
\begin{array}{cc}
\text { а) адсорбція: } & \text { b) десорбція: } \\
\left.c_{0}(t, z)\right|_{z=0}=c_{\text {in }}, & \left.c_{0}(t, z)\right|_{z=0}=c_{\text {in }}(t), \\
\left.\frac{\partial}{\partial z} c_{0}(t, z)\right|_{z=\infty}=0, & \left.\frac{\partial}{\partial z} c_{0}(t, z)\right|_{z=\infty}=0, \\
\left.T_{0}(t, z)\right|_{z=0}=T_{i n},\left.\frac{\partial}{\partial z} T_{0}(t, z)\right|_{z=\infty}=0, & \left.T_{0}(t, z)\right|_{z=0}=T_{i n}(t),\left.\frac{\partial}{\partial z} T_{0}(t, z)\right|_{z=\infty}=0 .
\end{array}
$$

Задача $A_{n} ; n=\overline{1, \infty}$ (n-го наближення з нульовими початковими та крайовими умовами): побудувати в області $D$ обмежений розв'язок системи рівнянь

$$
\begin{gathered}
\frac{\partial c_{n}(t, \mathrm{z})}{\partial t}+\frac{\partial a_{n}(t, \mathrm{z})}{\partial t}+u \frac{\partial c_{n}}{\partial z}=D_{\text {inter }} \frac{\partial^{2} c_{n}}{\partial z^{2}} \\
-H \frac{\partial T_{n}(t, z)}{\partial t}-u h_{g} \frac{\partial T_{n}}{\partial z}-Q \frac{\partial a_{n}}{\partial t}-\mathrm{X}^{2} T_{n}+\Lambda \frac{\partial^{2} T_{n}}{\partial z^{2}}=0
\end{gathered}
$$




$$
\frac{\partial a_{n}}{\partial t}=\beta\left(c_{n}-\gamma a_{n}-\sum_{i=0}^{n-1}\left(a_{i}(t, \mathrm{z})\left(a_{n-1-i}(t, \mathrm{z})-\frac{R}{\Delta H} T_{n-1-i}(t, \mathrm{z})\right)\right)\right) .
$$

Задача $A_{0} \in$ лінійною щодо нульового наближення $c_{0}, a_{0}, T_{0}$. Задача $A_{n} ; n=\overline{1, \infty}$ лінійна щодо $n$-го наближення та нелінійна щодо всіх попередніх $i$ наближень, $i=\overline{0, n-1}$.

Побудуємо аналітичні розв'язки задач $A_{0}$ та $A_{n} ; n=\overline{1, \infty}$ використовуючи операційний метод Гевісайда $[17 ; 18]$. Застосувавши по часовій змінній $t$ до задач (18)-(25) та (26)-(28) інтегральний оператор прямого перетворення Лапласа, одержимо наступну систему крайових задач в зображеннях за Лапласом [17]:

Задача $A_{0}^{*}$ : побудувати в області $D^{*}=\{z \in(0, \infty)\}$ обмежений розв'язок системи рівнянь:

$$
\begin{aligned}
& \frac{d^{2} c_{0}^{*}(p, \mathrm{z})}{d z^{2}}-u_{1} \frac{d c_{0}^{*}}{d z}-q_{1}^{2}(p) c_{0}^{*}=-\mathcal{F}_{c_{0}}^{*}(p), \\
& \frac{d^{2}}{d z^{2}} T_{0}^{*}-u_{2} \frac{d}{d z} T_{0}^{*}-q_{2}^{2}(p) T_{0}^{*}=-\mathcal{F}_{T_{0}}^{*}(p), \\
& a_{0}^{*}(p, \mathrm{z})=\beta \frac{1}{p+\beta \gamma} c_{0}^{*}(p, \mathrm{z}) .
\end{aligned}
$$

Крайові умови:

$$
\begin{array}{ll}
\text { а) адсорбція: } & \text { b) десорбція: } \\
\left.c_{0}^{*}(p, z)\right|_{z=o}=c_{i n}^{*}(p), & \left.\frac{d}{d z} c_{0}^{*}(p, z)\right|_{z=\infty}=0, \\
\left.\frac{\partial}{\partial z} c_{0}^{*}(p, z)\right|_{z=\infty}=0, & \left.c_{0}^{*}(p, z)\right|_{z=o}=\frac{1}{p} c_{i n}, \\
\left.T_{0}^{*}(p, z)\right|_{z=0}=\frac{1}{p} T_{i n},\left.\frac{\partial}{\partial z} T_{0}^{*}(p, z)\right|_{z=\infty}=0,\left.\frac{\partial}{\partial z} T_{0}^{*}(p, z)\right|_{z=\infty}=0, \\
\end{array}
$$

Задача $A_{n}^{*} ; n=\overline{1, \infty}$ : побудувати в області $D^{*}=\{z \in(0, \infty)\}$ обмежений розв'язок системи рівнянь:

$$
\begin{aligned}
& \frac{d^{2} c_{n}^{*}}{d z^{2}}-u_{1} \frac{d c_{n}^{*}}{d z}-q_{1}^{2}(p) c_{n}^{*}=-\mathcal{F}_{c_{n}}{ }^{*}(p, \mathrm{z}), \\
& \frac{d^{2}}{d z^{2}} T_{n}^{*}-u_{2} \frac{d}{d z} T_{n}^{*}-q_{2}^{2}(p) T_{n}^{*}=-\mathcal{F}_{T_{n}}{ }^{*}(p, z),
\end{aligned}
$$




$$
a_{n}^{*}(p, z)=\beta \frac{1}{p+\beta \gamma}\left(c_{n}^{*}-\left(\sum_{i=0}^{n-1} a_{i} a_{n-1-i}\right)^{*}(p, z)\right) \text {, }
$$

де $u_{1}=\frac{u}{D_{\text {inter }}}, \quad q_{1}^{2}(p)=\frac{p(p+\beta(\gamma+1))}{D_{\text {inter }}(p+\beta \gamma)}, \quad q_{2}^{2}(p)=\frac{H p+\mathrm{X}^{2}}{\Lambda}$, $\mathcal{F}_{c_{0}}^{*}=\frac{c_{0}^{0}}{D_{\text {inter }}}, \quad \mathcal{F}_{T_{0}}^{*}(p, z)=\frac{1}{\Lambda}\left(H T_{0}^{0}-Q \beta\left(1-\frac{\beta \gamma}{p+\beta \gamma}\right) c_{0}^{*}(p, z)\right)$, $\mathcal{F}_{c_{n}}{ }^{*}(p, z)=\frac{\beta}{D_{\text {inter }}}\left(1-\frac{\beta \gamma}{p+\beta \gamma}\right)\left(\sum_{i=0}^{n-1} a_{i}\left(a_{n-1-i}-\frac{R}{\Delta H} T_{n-1-i}\right)\right)^{*}(p, z)$, $\mathcal{F}_{T_{n}}{ }^{*}(p, z)=-\frac{Q \beta}{\Lambda}\left(1-\frac{\beta \gamma}{p+\beta \gamma}\right)\left(c_{n}^{*}(p, z)-\left(\sum_{i=0}^{n-1} a_{i}\left(a_{n-1-i}-\frac{R}{\Delta H} T_{n-1-i}\right)\right)^{*}(p, z)\right)$, $c^{*}(p, z)=\int_{0}^{\infty} c(t, z) \mathrm{e}^{-p t} d t \equiv L[c], a^{*}(p, Z)=\int_{0}^{\infty} a(t, z) \mathrm{e}^{-p t} d t \equiv L[a], T^{*}(p, z)=\int_{0}^{\infty} T(t, z) \mathrm{e}^{-p t} d t \equiv L[T]$.

Розв'язок неоднорідної крайової задачі $A_{0}$. Побудуємо розв'язок неоднорідної крайової задачі $A_{0}^{*}$ (використовуючи метод Коші) $[11 ; 18 ; 19]$ :

$$
\begin{gathered}
c_{0}^{*}(p, z)=\left(p c_{i n}^{*}(p)\right) e^{\frac{u_{1}}{2} z} \frac{e^{-\omega_{1}(p) z}}{p}+c_{0}^{0} \frac{\gamma}{\gamma+1}\left(\frac{1}{p}+\frac{1}{p+\beta(\gamma+1)}-\frac{\gamma+1}{\gamma} e^{\frac{u_{1}}{2} z} \frac{e^{-\omega_{1}(p) z}}{p}\right)+ \\
+c_{0}^{0} \frac{\beta}{(p+\beta(\gamma+1))} \frac{e^{-\omega_{1}(p) z}}{p} e^{\frac{u_{1}}{2} z} \\
T_{0}^{*}(p, z)=p T_{i n}^{*}(p) \frac{e^{\left(\frac{u_{2}}{2}-\omega_{2}(p)\right) z}}{p}+\int_{0}^{\infty} e^{\frac{u_{2}}{2}(z-\xi)}\left(\frac{e^{-\omega_{2}(p) z-\xi \mid}}{2 \omega_{2}(p)}-\frac{e^{-\omega_{2}(p)(z+\xi)}}{2 \omega_{2}(p)}\right) \mathcal{F}_{T_{0}}^{*}(p, z, \xi) d \xi ;
\end{gathered}
$$

де

$$
\begin{gathered}
\omega_{1}(p)=\left(\frac{u_{1}^{2}}{4}+q_{1}^{2}(p)\right)^{1 / 2}, \omega_{2}(p)=\left(\frac{u_{2}^{2}}{4}+q_{2}^{2}(p)\right)^{1 / 2}, \\
\operatorname{Re} \omega_{1}>0, \operatorname{Re} \omega_{2}>0 .
\end{gathered}
$$

Застосовуючи інтегральний оператор оберненого перетворення Лапласа $L^{-1}[\ldots *(p, \mathrm{z})]=\frac{1}{2 \pi i} \int_{\sigma_{0}-i \infty}^{\sigma_{0}+{ }^{+\infty}} \ldots *^{*}(p, \mathrm{z}) e^{p t}$ до формул (38), (39), отримуємо на основі [10; 11] їх оригінали, що формують аналітичний розв'язок задачі $A_{0}$ нульового наближення: залежність концентра- 
ції адсорбату в газовій фазі (міжчастинковий простір) температури та концентрації адсорбату в частинках нанопористого цеоліту вздовж координати шару та в часі для циклів адсорбції та десорбції

$$
\begin{aligned}
& c_{0}(t, z)=c_{i n}(0) e^{\frac{u}{2 D_{\text {inter }}} z} \Phi_{c}^{0}(t, z)+e^{\frac{u}{2 D_{\text {inter }}} z} \int_{0}^{t} \frac{d}{d \tau} c_{i n}(\tau) \Phi_{c}^{0}(t-\tau, z) d \tau+ \\
& +c_{0}^{0} \frac{\gamma}{1+\gamma}\left(1+\frac{1}{\gamma} e^{-\beta(\gamma+1) t}-\frac{\gamma+1}{\gamma} e^{\frac{u}{2 D_{\text {inter }}} z} \Phi_{c}^{0}(t, z)\right)+\beta c_{0}^{0} e^{\frac{u}{2 D_{\text {inter }}} z} \int_{0}^{t} e^{-\beta(\gamma+1)(t-s)} \Phi_{c}^{0}(\tau, z) d \tau \\
& T_{0}(t, \mathrm{z})=T_{i n}(0) \Phi_{T}^{0}(t, z)+\int_{0}^{t} \frac{d}{d \tau} T_{i n}(\tau) \Phi_{T}^{0}(t-\tau, z)+ \\
& +\frac{1}{\Lambda} \int_{0}^{t} \int_{0}^{\infty}\left[H T_{0}^{0} \mathcal{H}_{T}(t-\tau ; z, \xi)-Q \beta\left(\mathcal{H}_{T}(t-\tau, z, \xi)-\beta \gamma \int_{0}^{t-\tau} e^{-\beta \gamma(t-\tau-s)} \mathcal{H}_{T}(\tau-s ; z, \xi) d s\right) c_{0}^{*}(p, \xi)\right] d \xi d \tau \\
& a_{0}(t, z)=\beta \int_{0}^{t} e^{-\gamma \beta(t-\tau)} c_{0}(\tau, z) d \tau
\end{aligned}
$$

Тут

$$
\begin{aligned}
& \Phi_{c}^{0}(t, z)==\frac{1}{\pi} \int_{0}^{\pi} e^{-\varphi_{1}(v) z} \frac{\sin \left(v t-z \varphi_{2}(v)^{2}\right)}{v} d v+e^{-\frac{u}{2 D_{\text {inter }}} z}, \\
& \Phi_{c}(t, \mathrm{z})=\frac{1}{2 \pi} \int_{0}^{\infty} \frac{\varphi_{1}(v) \cos \left(v t-\varphi_{2}(v) \mathrm{z}\right)+\phi_{2}(v) \sin \left(v t-\varphi_{2}(v) \mathrm{z}\right)}{\left(\Gamma_{1}^{2}(v)+v^{2} \Gamma_{2}^{2}(v)\right)^{1 / 2}} d v \text {. } \\
& \Phi_{T}^{0}(t, z)=\frac{1}{\pi} \int_{0}^{\infty} e^{-\phi_{1}(v) z} \frac{\sin \left(v t-z \phi_{2}(v)^{2}\right)}{v} d v+e^{-\frac{u}{2 D_{\text {mer }}} z} \\
& \Phi_{T}(t, \mathrm{z})=\frac{1}{2 \pi} \int_{0}^{\infty} \frac{\phi_{1}(v) \cos \left(v t-\phi_{2}(v) \mathrm{z}\right)+\phi_{2}(v) \sin \left(v t-\phi_{2}(v) \mathrm{z}\right)}{\left(\Gamma_{T_{1}}^{2}(v)+v^{2} \Gamma_{T_{2}}^{2}(v)\right)^{1 / 2}} d v \text {, } \\
& \text { де } \varphi_{1,2}(v)=\left[\frac{\left(\Gamma_{1}^{2}(v)+v^{2} \Gamma_{2}^{2}(v)\right)^{1 / 2} \pm \Gamma_{1}^{2}(v)}{2}\right]^{1 / 2}, \Gamma_{1}(v)=\frac{u^{2}}{4 D_{\text {inter }}^{2}}+\frac{v^{2} \beta}{D_{\text {inter }}^{2}\left(v^{2}+\beta^{2} \gamma^{2}\right)} \text {, }
\end{aligned}
$$

$$
\begin{array}{cc}
\Gamma_{2}(v)=\frac{v^{3}+v \beta^{2}(\gamma+1) \gamma}{D_{\text {inter }}\left(v^{2}+\beta^{2} \gamma^{2}\right)}, & \phi_{1,2}(v)=\left[\frac{\left(\Gamma_{T_{1}}^{2}(v)+v^{2} \Gamma_{T_{2}}^{2}(v)\right)^{1 / 2} \pm \Gamma_{T_{1}}^{2}(v)}{2}\right]^{1 / 2}, \\
\Gamma_{T_{1}}(v)=\frac{u^{2}+4 \Lambda X^{2}}{4 \Lambda^{2}}, & \Gamma_{T_{2}}(v)=\frac{H v}{\Lambda} .
\end{array}
$$

При переході до оригіналу Лапласа в формулі (41) перетворен- 
ня першої компоненти здійснюється з використанням інтеграла Дюамеля [17], де $\mathcal{H}_{T}(\tau ; z, \xi)=e^{-\frac{u_{2}}{2}(z-\xi)}\left(\Phi_{T}(\tau,|z-\xi|)-\Phi_{T}(\tau, z+\xi)\right)$.

Розв'язок неоднорідної крайової задачі $A_{n} ; n=\overline{1, \infty}$. Розв'язки задач $A_{n}^{*} ; n=\overline{1, \infty} \in$ функціями [11]:

$$
\begin{gathered}
c_{n}^{*}(p, \mathrm{z})=\int_{0}^{\infty} e^{\frac{u_{1}}{2}(z-\xi)}\left(\frac{e^{-\omega_{1}(p)|z-\xi|}}{2 \omega_{1}(p)}-\frac{e^{-\omega_{1}(p)(z+\xi)}}{2 \omega_{1}(p)}\right) \mathcal{F}_{c_{n}}{ }^{*}(p, \xi) d \xi, \\
T_{n}^{*}(p, z)=\int_{0}^{\infty} e^{\frac{u_{2}}{2}(z-\xi)}\left(\frac{e^{-|z-\xi| \omega_{2}(p)}}{2 \omega_{2}(p)}-\frac{e^{-(z+\xi) \omega_{2}(p)}}{2 \omega_{2}(p)}\right) \mathcal{F}_{T_{n}}^{*}(p, \xi) d \xi, \\
a_{n}^{*}(p, \mathrm{z})=\frac{\beta}{p+\gamma \beta}\left[c_{n}^{*}(p, \mathrm{z})-\left(\sum_{i=0}^{n-1} a_{i}\left(a_{n-1-i}-\frac{R}{\Delta H} T_{n-1-i}\right)\right)^{*}(p, \mathrm{z})\right] .
\end{gathered}
$$

Підставляючи значення $\mathcal{F}_{c_{n}}{ }^{*}(p, \xi)$ і $\mathcal{F}_{T_{n}}^{*}(p, \xi)$ відповідно в рівняння. (43), (44) і застосовуючи інтегральний оператор оберненого перетворення Лапласа до формул (43)-(45), отримаємо на основі [10; 11] їх оригінали, які формують аналітичний розв'язок задачі $A_{n} ; n=\overline{1, \infty}$, що описує часово-просторові розподіли п-го наближення адсорбційних концентрацій у газової фази $c_{n}(t, z)$, температури $T_{n}(t, z)$ та адсорбції (концентрація адсорбату в нанопорах частинок) $a_{n}(t, z)$ :

$c_{n}(t, \mathrm{z})=\frac{\beta}{D_{\text {inter }}} \int_{0}^{\tau} \int_{0}^{\infty}\left[\mathcal{H}_{c}(t-\tau ; z, \xi)-\beta \gamma \int_{0}^{t-\tau} e^{-\beta \gamma \gamma \tau-s)} \mathcal{H}_{c}(\mathrm{~s} ; z, \xi) d s\right]\left(\sum_{i=0}^{n-1} a_{i}\left(a_{n-1-i}-\frac{R}{\Delta H} T_{n-1-i}\right)\right)(\tau, \xi) d \xi d \tau ;$ (46)

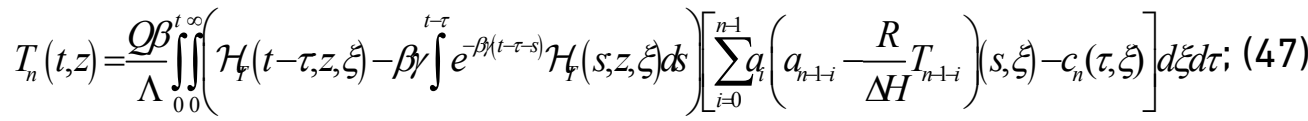
$a_{n}(t, \mathrm{z})=\beta \int_{0}^{t} e^{-\beta \gamma(t-\tau)}\left(c_{n}(\tau, \mathrm{z})-\sum_{i=0}^{n-1} a_{i}\left(a_{n-1-i}-\frac{R}{\Delta H} T_{n-1-i}\right)(\tau, \mathrm{z})\right) d \tau$, де $\mathcal{H}_{c}(\tau ; z, \xi)=e^{-\frac{u_{1}}{2}(z-\xi)}\left(\Phi_{c}(\tau,|z-\xi|)-\Phi_{c}(\tau, z+\xi)\right)$.

3. Комп'ютерне моделювання. Аналіз розподілів концентрації адсорбтиву в газовій фазі і нанопорах цеоліту та температур Метою комп'ютерного моделювання було вивчення можливос- 
тей моделі, запропонованої для її подальшого використання в технологіях очищення викидів вуглецю в атмосферу енергетичними та транспортними об'єктами (пропан, $\mathrm{CO}_{2}$ та інші продукти згоряння). Сьогодні це один із ключових способів вирозв'язок проблем глобального потепління та створення безпечної енергетичної стратегії [2].

В якості адсорбату було обрано пропан, обсяг якого охоплює близько 30\% загального потоку газу, що виходить з двигуна автомобіля протягом першої хвилини його експлуатації [3]. Використовуючи розроблену теорію, проведено моделювання та обчислення концентраційних залежностей неізотермічних кривих адсорбції та десорбції в нанопорошкових каталітичних пластах. Розрахунки проводились для експериментального зразка [13]. Геометричні розміри зразка: довжина $-l=1,5 \cdot 10^{-2} \mathrm{M}$, радіус $R=0,45 \cdot 10^{-2}$ м. Фізичні параметри цеоліту взяті з [10; 13].

3 використанням розвиненої в даній статті теорії, застосовуючи залежності (40), (46) виконувались розрахунки концентрацій адсорбату у газоподібній фазі. На рис. $1, a, b, c, d$ приведено залежності розрахованих величин концентрації $c=c(t, z)$ від часу для фіксованих значень геометричних розмірів досліджуваного зразка та фіксованих значень температури $20^{\circ} \mathrm{C}, 50^{\circ} \mathrm{C}, 100^{\circ} \mathrm{C}, 300^{\circ} \mathrm{C}$, за якої відбуваються адсорбативні процеси.

Як видно з розрахованих залежностей від часу $t$, їх загальний характер є якісно однаковим: для кожного з значень $z / z_{0}$ значення концентрації адсорбату $c(t) / c_{0}$ зростають. При цьому на графіках рис. 1, a, b, с можна спостерігати незначне зменшення концентрації адсорбату, а далі його концентрація збільшується, виходячи на насичення. Слід зауважити, що з ростом температури виявлений ефект стає все менш вираженим. Поява цього ефекту пов'язана з тим, що при малій температури молекули відштовхуються одна від одної, як це видно зі співвідношення Ленарда-Джонса, що спричиняє часткове вивільнення нанопорами адсорбату частини адсорбованих молекул. 3 ростом ж температури сили відштовхування між молекулами слабшають, і згаданих ефект стає слабовираженим. Також слід зауважити, що швидкість росту концентрації адсорбату у газовій фазі сильно залежить від температури: вона швидко зростає з їі ростом. 

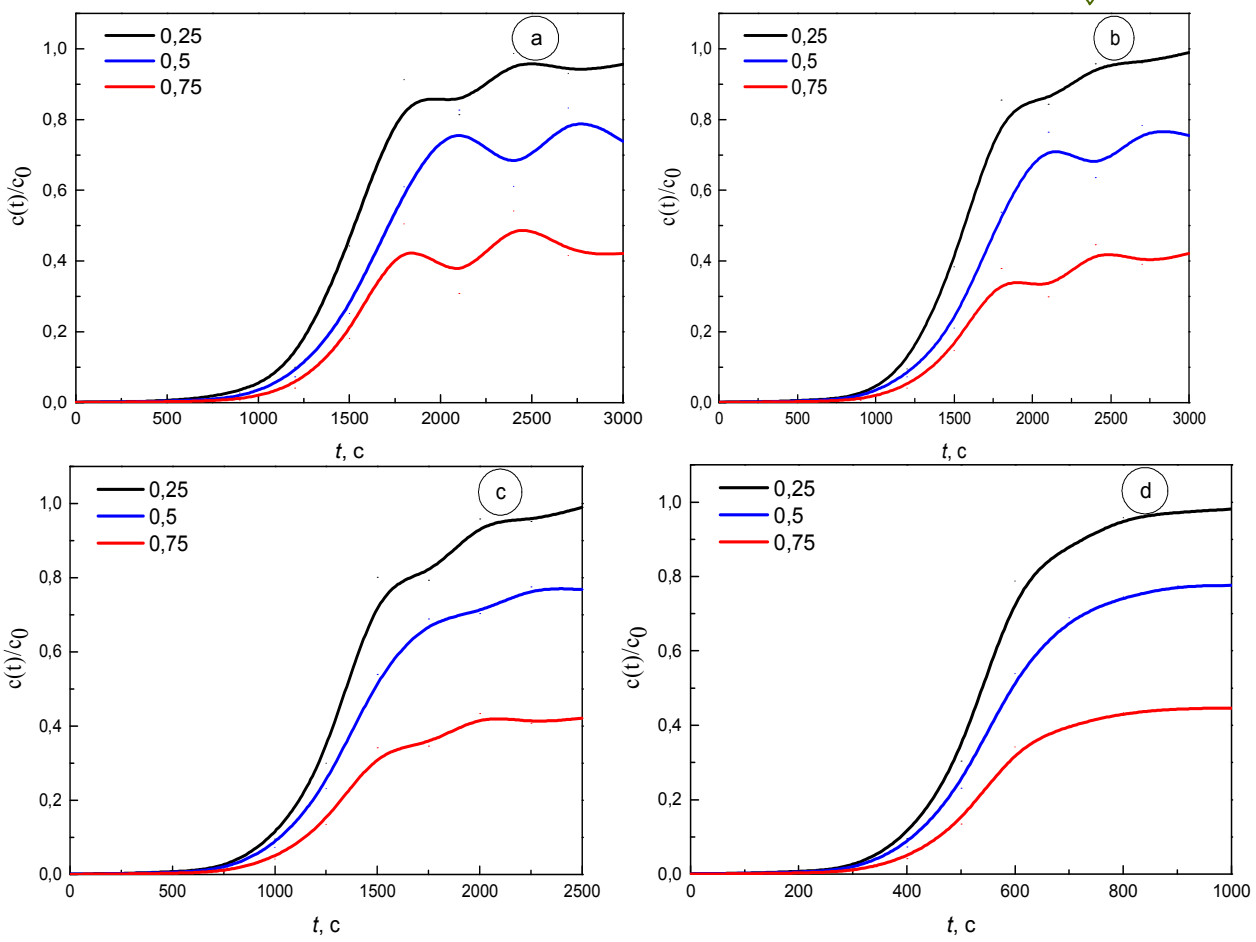

Рис. 1. Залежності концентрації адсорбату в газовій фазі $c=c(t, z)$ за фіксованими значеннями $z\left(z / z_{0}=0,25 ; 0,5 ; 0,75\right)$, розрахованими при температурі $20^{\circ} \mathrm{C}$ (а), $50^{\circ} \mathrm{C}$ (б), $100^{\circ} \mathrm{C}$ (c), $300^{\circ} \mathrm{C}$ (d)

На рис. 2, a, b, c, d приведено результати розрахунку концентрації адсорбованої речовини з газової фази у нанопорах адсорбату. Розрахунки виконано у залежності від часу $t$ для аналогічних геометричних параметрів досліджуваного зразка та значень $z$ та температури, при яких отримано залежності, приведені на рис. 2, a, b, c, d.

Як видно з аналізу приведених на рис. 2, a, b, c, d залежностей, їх графіки мають одну характерну особливість: у кожному з графіків зі зміною часу формується похиле плато, пов'язане зі зростанням концентрації у порах адсорбенту. Після досягнення ж максимуму у кожній з концентраційних залежностей формується вертикальна стінка, що з фізичної точки зору визначає діапазон часу, за який нанопори втрачають захоплений леткий адсорбат. Слід також зауважити, що попри схожість отриманих залежностей, з ростом температури концентрація $a(t)$ у порах адсорбенту різко зменшується. Крім того, максимуми кожної з залежностей з ростом температури зміщуються у шкалі часу вправо, проте, як видно з графіківв зменшення концентрації відбувається протягом також самого інтервалу часу. 

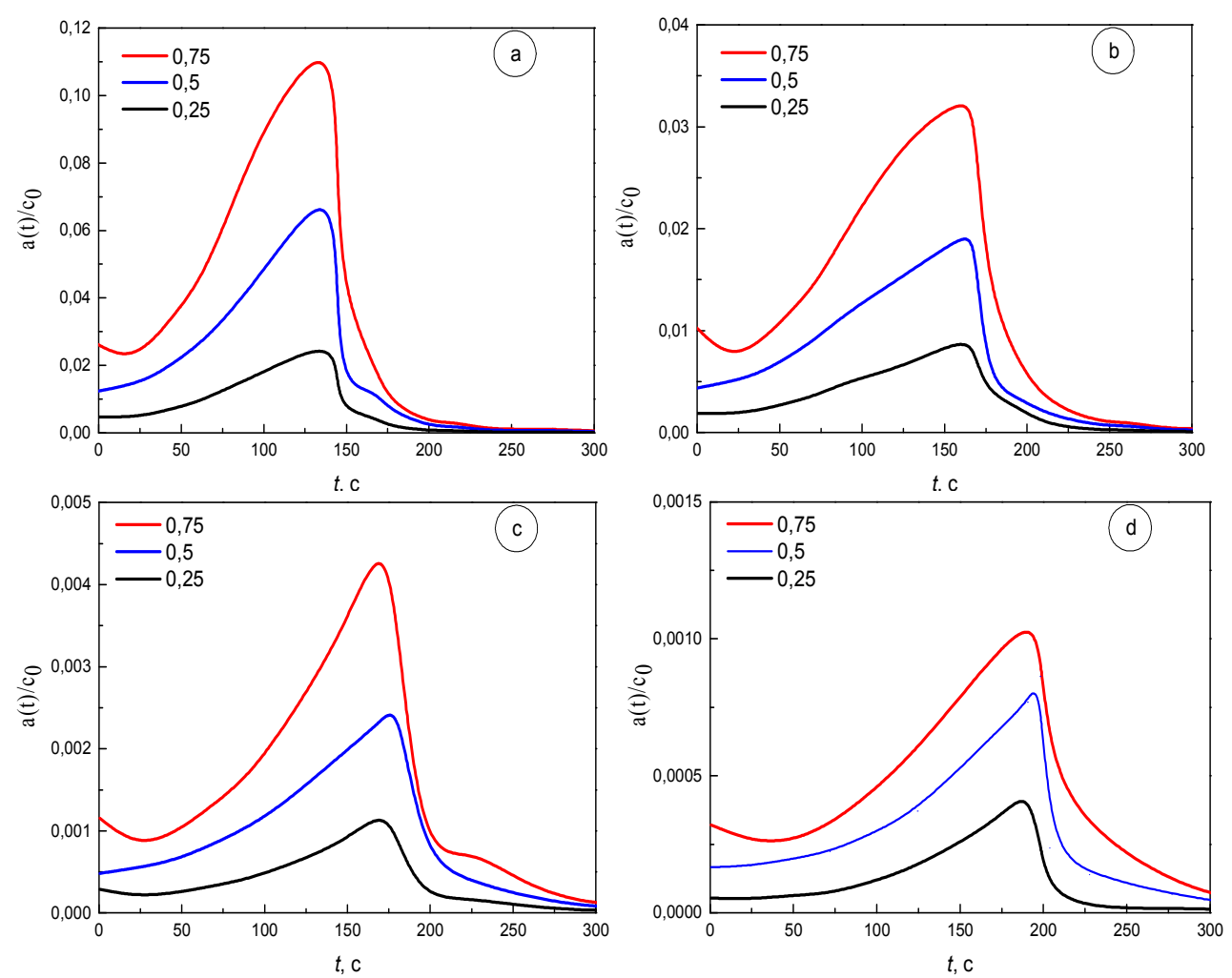

Рис. 2. Залежність концентрації в порах адсорбенту $a=a(t, z)$ при фіксованих значеннях $z\left(z / z_{0}=0,25 ; 0,5 ; 0,75\right)$, розрахованих при температypi $20^{\circ} \mathrm{C}\left(\right.$ а), $50^{\circ} \mathrm{C}$ (б), $100^{\circ} \mathrm{C}$ (с), $300^{\circ} \mathrm{C}$ (г)

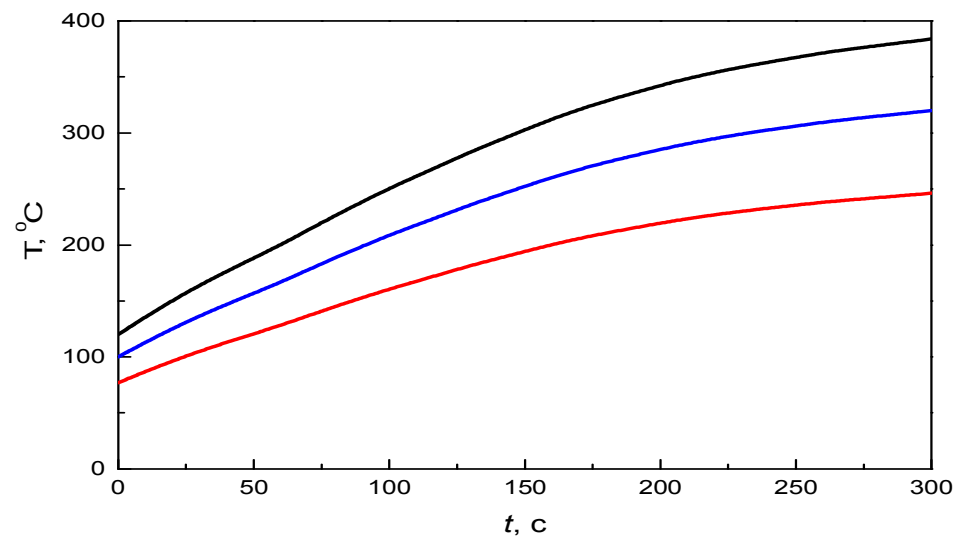

Рис. 3. Залежність температури всередині зразка $T=T(t, z)$ від часу за фіксованими значеннями $z\left(z / z_{0}=0,25 ; 0,5 ; 0,75\right)$

Також, як видно з рис. 3 на процес істотно впливає зростання температури усередині досліджуваного зразка. Співставляючи гра- 
фіки рис. 2, a, b, c, $d$ і рис. 3, видно, що процес поглинання нанопорами адсорбенту відбувається в проміжку від 50 с до 200 с, при цьому температура всередині досліджуваного зразка змінюється в середньому від $100^{\circ} \mathrm{C}$ до $320^{\circ} \mathrm{C}$.

На рис. 4 продемонсторвані криві, що відображають зміну концентрації адсорбату в газовій фазі від швидкості вхідного потоку і температури при фіксованих значеннях тривалості адсорбції $\tau=360 \mathrm{c}$. В залежності від зміни швидкості газового потоку можна відслідковувати та оцінювати робочі зони максимального та мінімального поглинання адсорбату порами нанопористих частинок і у такий спосіб регулювати тривалість стадії адсорбції, забезпечивши високий ступінь поглигання адсорбату нанопорами.

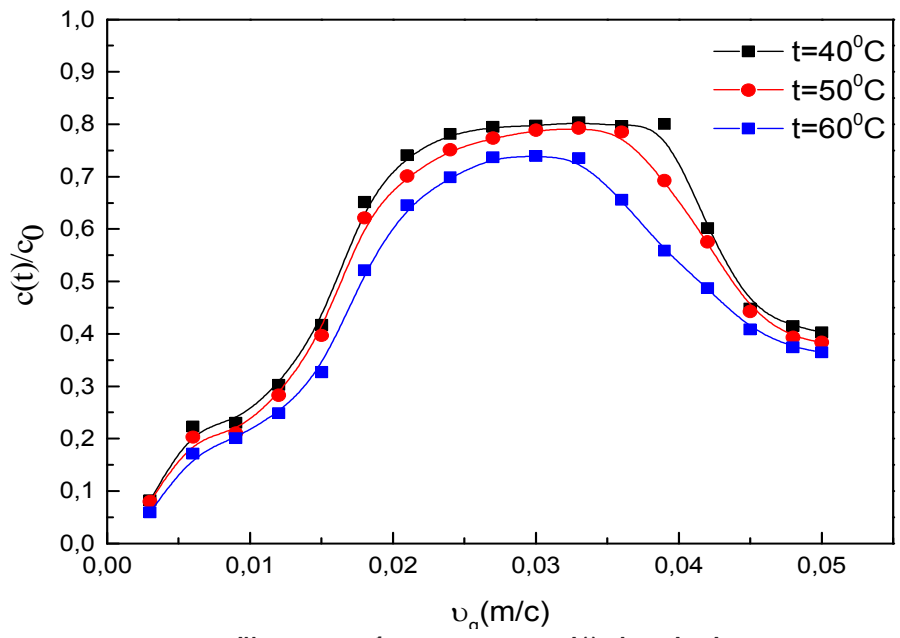

Рис. 4. Зміна концентрації адсорбату в газовій фазі від швидкості вхідного потоку і температури при фіксованих значеннях тривалості адсорбції

$$
\mathrm{T}=360 \text { c i } z\left(z / z_{0}=0,25 ; 0,5 ; 0,75\right)
$$

Висновки. Розроблені високопродуктивні методи і обчислювальні технології моделювання неізотермічних адсорбції і десорбції в нанопористих каталізаторах для класів нелінійних ізотерм адсорбції газів в нанопористих цеоліт-системах. Обґрунтовані і розвинуті підходи побудови узагальнених нелінійних рівнянь рівноваги адсорбції в рамках полімолекулярної теорії адсорбційної рівноваги Ленгмюра, на випадки неізотермічної циклічної адсорбції-десорбції шляхом включення в них компонентів впливу температури середовища та енергії активації. На їх основі побудовані нові нелінійні математичні моделі, що включають балансові рівняння адсорбції/десорбції з урахуванням взаємодії нано- і макропотоків, дисперсійних сил ЛенардаДжонса, швидкості і температури газового потоку. Реалізовані ефек- 
тивні схеми лінеаризації нелінійних моделей на основі підходу Ландау щодо розкладу в ряд функції рівноваги в околі точки температури фазового переходу та малого параметру. Побудовані високошвидкісні аналітичні та числові розв'язки математичних моделей з використанням операційного методу Гевісайда, здійснена їх алгоритмічна і програмна реалізація, що дозволило здійснити ефективне розпаралелювання обчислювальних процесів та підвищити швидкодію обчислень в цілому.

1. Unger N., Bond T. C., Wang J. S., Koch D. M., Menon S., Shindell D. T., Bauer S. Attribution of climate forcing to economic sectors. Proc. Natl. Acad. Sci. 2010. 107(8). 3382-3387. 2. Euro 5 and Euro 6 standards: reduction of pollutant emissions from light vehicles. Availableat: Europa.eu/legislation_summaries/environment/air_pollution/l28186_es.htm (2010). 3. Puertolas B., Navarro M. V., Lopez J. M., Murillo R., Mastral A. M., Garcia T. Modelling the heat and mass transfers of propane onto a ZSM-5 zeolite. Separation and Purification Technology 86. 2012. 127-136. 4. Michuta 0., Martuniuk P., Ostapchuk 0., Tsvetkova T. On non-isothermal soil water flow considering sorption effect. Journal of Heat and Mass Transfer. 2019. Vol. 18 (1), 181-192. 5. Бомба А. Я., Булавацький В. М., Скопецький В. В. Нелінійні математичні моделі процесів геогідродинаміки. Київ: Наукова думка, 2007. 308 c. 6. Petryk M., Fraissard J., Leclerc S., Canet D. Modeling of gas transport in a microporous solid using a slice selection procedure: Application to the diffusion of benzene in ZSM5. Catalysis Today, Elsevier. 2008. Vol. 139(3). 234-240. 7. Lecler S., Petryk M., Canet D., Fraissard J. Competitive Diffusion of Gases in a Zeolite Using Proton NMR and Sclice Selection Procedure. Catalysis Today, Elsevier B.V. 2012. Vol. 187(1). 104-107. 8. Petryk M., Leclerc S., Canet D., Sergienko I. V., Deineka V. S., Fraissard J. The Competitive Diffusion of Gases in a zeolite bed: NMR and Slice Procedure, Modelling and Identification of Parameters. The Journal of Physical Chemistry C. ACS. 2015. 119 (47). 26519-26525. 9. Sergienko I. V., Petryk M. R., Leclerk S., Fraissard J. High productivity methods of identification of competitive diffusion parameters in heterogeneous media of nanoporous particles. Cybernetics and Systems Analysis. 2015. 51(4), 529-546. 10. Petryk M., Khimitch A., Petryk M. M. Simulation of Adsorption and Desorption of Hydrocarbons in Nanoporous Catalysts of Neutralization Systems of Exhaust Gases Using Nonlinear Langmuir Isotherm. Journal of Automation and Information Sciences. 2018. Vol. 50 (10). 18-33. 11. Математичне моделювання теплопереносу та адсорбції вуглеводнів в нанопористих цеолітних каталізаторах систем нейтралізації відпрацьованих газів / Петрик М. Р., Хіміч О. М., Бойко І. В., Михалик Д. М., Петрик М. М., Ковбашин В. І. Національна академія наук України Ін-т кібернетики ім. B.М. Глушкова, 2017. 280 с. 12. Langmuir I. J. Am. Chem. Soc. 54, 2798 (1932). 13. Kärger J., Ruthven D., Theodorou D. Diffusion in Nanoporous Materials. Hoboken: John Wiley \& Sons, 2012. 660 p. 14. Kärger J. and Ruthven D. Diffusion in Zeolites and Other Microporous Solids, John Wiley \& Sons, New York, 1992. 605 p. 15. Landau L. To the theory of phase transitions. 
I. ZPhys. Ztshr Sow, 1937, Bd. 7. S. 19. 16. Prudnikov A. P., Brichkov Yu. A. Marychev 0. I. Integrals and series. Additional chapters, Nauka, Moscow, 1986. 800 p. 17. Lavrentiev M. A., Shabat B. V. Methods of theory of functions of a complex variable. M. : Nauka, 1973. 736 p. 18. Petryk M., Khimitch A., Petryk M.M., Fraissard J. Experimental and computer simulation studies of dehydration on microporous adsorbent of natural gas used as motor fuel. Fuel. 2019. Vol. 239. 1324-1330. 19. Petryk M., Ivanchov M., Leclerc S., Canet D., Fraissard J. Competitive Adsorption and Diffusion of Gases in a Microporous Solid. Zeolites - New Challenges. IntecOpen London, UK. 2019. P. 1-23. URL: https://www.intechopen.com/online-first/competitive-adsorption-anddiffusion-of-gases-in-a-microporous-solid (дата звернення: 24.10.2019).

\section{REFERENCES:}

1. Unger N., Bond T. C., Wang J. S., Koch D. M., Menon S., Shindell D. T., Bauer S. Attribution of climate forcing to economic sectors. Proc. Natl. Acad. Sci. 2010. 107(8). 3382-3387. 2. Euro 5 and Euro 6 standards: reduction of pollutant emissions from light vehicles. Availableat: Europa.eu/legislation_summaries/environment/air_pollution/l28186_es.htm (2010). 3. Puertolas B., Navarro M. V., Lopez J. M., Murillo R., Mastral A. M., Garcia T. Modelling the heat and mass transfers of propane onto a ZSM-5 zeolite. Separation and Purification Technology 86. 2012. 127-136. 4. Michuta O., Martuniuk P., Ostapchuk 0., Tsvetkova T. On non-isothermal soil water flow considering sorption effect. Journal of Heat and Mass Transfer. 2019. Vol. 18 (1), 181-192. 5. Bomba A. Ya., Bulavatskyi V. M., Skopetskyi V. V. Neliniini matematychni modeli protsesiv heohidrodynamiky. Kyiv : Naukova dumka, 2007. 308 c. 6. Petryk M., Fraissard J., Leclerc S., Canet D. Modeling of gas transport in a microporous solid using a slice selection procedure: Application to the diffusion of benzene in ZSM5. Catalysis Today, Elsevier. 2008. Vol. 139(3). 234-240. 7. Lecler S., Petryk M., Canet D., Fraissard J. Competitive Diffusion of Gases in a Zeolite Using Proton NMR and Sclice Selection Procedure. Catalysis Today, Elsevier B.V. 2012. Vol. 187(1). 104-107. 8. Petryk M., Leclerc S., Canet D., Sergienko I. V., Deineka V. S., Fraissard J. The Competitive Diffusion of Gases in a zeolite bed: NMR and Slice Procedure, Modelling and Identification of Parameters. The Journal of Physical Chemistry C. ACS. 2015. 119 (47). 26519-26525. 9. Sergienko I. V., Petryk M. R., Leclerk S., Fraissard J. High productivity methods of identification of competitive diffusion parameters in heterogeneous media of nanoporous particles. Cybernetics and Systems Analysis. 2015. 51(4), 529-546. 10. Petryk M., Khimitch A., Petryk M. M. Simulation of Adsorption and Desorption of Hydrocarbons in Nanoporous Catalysts of Neutralization Systems of Exhaust Gases Using Nonlinear Langmuir Isotherm. Journal of Automation and Information Sciences. 2018. Vol. 50 (10). 18-33. 11. Matematychne modeliuvannia teploperenosu ta adsorbtsii vuhlevodniv $v$ nanoporystykh tseolitnykh katalizatorakh system neitralizatsii vidpratsovanykh haziv / Petryk M. R., Khimich 0. M., Boiko I. V., Mykhalyk D. M., Petryk M. M., Kovbashyn V. I. Natsionalna akademiia nauk Ukrainy In-t 
kibernetyky im. V.M. Hlushkova, 2017. 280 c. 12. Langmuir I. J. Am. Chem. Soc. 54, 2798 (1932). 13. Kärger J.,Ruthven D., Theodorou D. Diffusion in Nanoporous Materials. Hoboken : John Wiley \& Sons, 2012.660 p. 14. Kärger J. and Ruthven D. Diffusion in Zeolites and Other Microporous Solids, John Wiley \& Sons, New York, 1992. 605 p. 15. Landau L. To the theory of phase transitions. I. ZPhys. Ztshr Sow, 1937, Bd. 7. S. 19. 16. Prudnikov A. P., Brichkov Yu. A. Marychev 0. I. Integrals and series. Additional chapters, Nauka, Moscow, 1986. 800 p. 17. Lavrentiev M. A., Shabat B. V. Methods of theory of functions of a complex variable. M. : Nauka, 1973. 736 p. 18. Petryk M., Khimitch A., Petryk M. M., Fraissard J. Experimental and computer simulation studies of dehydration on microporous adsorbent of natural gas used as motor fuel. Fuel. 2019. Vol. 239. 1324-1330. 19. Petryk M., Ivanchov M., Leclerc S., Canet D., Fraissard J. Competitive Adsorption and Diffusion of Gases in a Microporous Solid. Zeolites - New Challenges. IntecOpen London, UK. 2019. P. 1-23. URL: https://www.intechopen.com/online-first/competitiveadsorption-and-diffusion-of-gases-in-a-microporous-solid (data zvernennia: 24.10.2019).

Petryk M. R., Doctor of Physico-Mathematical Sciences, Professor, (Ternopil National Technical University named after Ivan Puliuy, Ternopil), Khimich 0. M., Corresponding Member of the National Academy of Science of Ukraine, Doctor of Physico-Mathematical Sciences, Professor (V.M. Glushkov Institute of Cybernetics, NASU, Kyiv), Boiko I. V., Candidate of Physico-Mathematical Sciences (Ph.D.), Associate Professor, Petryk 0. Yu. (Ternopil Ivan Puluj National Technical University, Ternopil)

\section{HIGH-PERFORMANCE CALCULATING METHODS AND TECHNOLOGIES OF SIMULATION OF NANOPOROUS SYSTEMS WITH FEEDBACK FOR ADSORPTION GAS CLEANING}

The problem of modeling non isothermal adsorption and desorption in nanoporous catalysts for nonlinear adsorption equilibrium of the Langmuir type in nanoporous zeolite systems is considered. Highly dispersed high-performance methods and computational technology models of non-isothermal adsorption and desorption in nanoporous catalysts for non-linear adsorbed gas classes in nanoporous zeolite systems. Turn around and move forward and encourage more nonlinear adsorption in the framework of the molecular theory of adsorption and in the middle of the second component. On the basis of these, new, non-linear mathematical models are encouraged, which include balancing adsorption / desorption systems with modalities of the nano- and macroflows, dispersion forces of the Lenard-Jones gas, 
and high-speed gas flow. Realization of effective schemes of linearization of nonlinear models based on the Landau approach should be placed in a series of Langmuir functions at a temperature transition point near a small parameter. Prompted by higher analytical and numerical analysis of mathematical models using the operational method of Heaviside, the algorithm is more efficient, and it is possible to significantly increase the efficiency of the process. The results of computational experiments of space-hourly concentration distributions of adsorbate in the gas phase and in the phase of micropores of the adsorbent in the deposits in the temperature and the speed of the gas flow are presented. Keywords: high-performance computations, nanoporous feedback systems, adsorption and desorption of gases, Langmuir adsorption equilibrium function, Heaviside operational method, Laplace integral transformation.

Петрик М. Р., д.ф.-м.н., профессор (Тернопольский национальный технический университет им. Ивана Пулюя, г. Тернополь), Химич О. М., чл.-кор. НАНУ, д.Ф.-М.н., профессор (Институт кибернетики им. В.М. Глушкова НАНУ, г. Киев), Бойко И. В., к.ф.-м.н., доцент, Петрик О. Ю. (Тернопольский национальный технический университет им. Ивана Пулюя, г. Тернополь)

ВЫСОКОПРОИЗВОДИТЕЛЬНЫЕ ВЫЧИСЛИТЕЛЬНЫЕ МЕТОДЫ И ТЕХНОЛОГИИ МОДЕЛИРОВАНИЯ НАНОПОРИСТЫХ СИСТЕМ С ОБРАТНОЙ СВЯЗЬЮ ДЛЯ АДСОРБЦИОННОЙ ОЧИСТКИ ГАЗОВ

Рассматривается задача моделирования неизотермической адсорбции и десорбции в нанопористых катализаторах для нелинейного адсорбционного равновесия типа Ленгмюра в нанопористых цеолит-системах. Предлагаются висопродуктивные методы операционного исчисления Хевисайда и преобразования Лапласа с использованием схем линеаризации на основе подхода Ландау по разложению в ряд функции равновесия Ленгмюра в окрестности точки температуры фазового перехода и малого параметра, позволяющие эффективное распараллеливание вычислений. Представленны результаты вычислительных экспериментов.

Ключевые слова: высокопроизводительные вычисления, нанопористые системы с обратными связями, адсорбция и десорбция газов, функция адсорбционного равновесия Ленгмюра, операционный метод Гевисайда, интегральное преобразование Лапласа. 